\title{
Factors influencing student nurse decisions to report poor practice witnessed while on placement
}

\author{
Ion R., Smith K., Nimmo S., Rice A.M., McMillan L.
}

This is the accepted manuscript (C) 2015, Elsevier

Licensed under the Creative Commons Attribution-NonCommercial-NoDerivatives 4.0 International http://creativecommons.org/licenses/by-nc-nd/4.0/

(cc) BY-NC-ND

The published article is available from doi:

http://dx.doi.org/10.1016/j.nedt.2015.02.006 


\section{Title: Factors influencing Student Nurse's Decisions to Report Poor Practice in the Practice Learning Environment.}

\section{Authors}

Ion R, Smith K, Nimmo S, Rice A, M and McMillan L

\section{Abstract}

\section{Background}

While it is commonly accepted that nursing care is generally of a good standard, it would be naïve to think that this is always the case. Over recent years concern about aspects of the quality of some nursing care has grown. In tandem with this, there is recognition that nurses do not always report poor practice. As future registrants, student nurses have a role to play in changing this culture. We know, however, relatively little about the factors that influence student decisions on whether or not to report. In the absence of a more nuanced understanding of this issue, we run the risk of assuming students will speak out simply because we say they should.

\section{Objectives}

To explore influences on student decisions about whether or not to report poor clinical practice which is a result of deliberate action and which is witnessed while on placement.

\section{Methods}

Qualitative interviews were conducted with thirteen pre-registration nursing students from the UK. Participants included both adult and mental health nurses with an age range from 20-47. Data were analysed to identify key themes. Category integrity and fit with data was confirmed by a team member following initial analysis. 


\section{Results}

Four themes emerged from the data. The first of these, 'I had no choice' described the personal and ethical drivers which influenced students to report. 'Consequences for self' and 'Living with ambiguity' provide an account of why some students struggle to report, while 'Being prepared' summarised arguments both for and against reporting concerns.

\section{Conclusion}

While there is a drive to promote openness in health care settings and an expectation that staff will raise concerns about quality of care, the reality is that the decision to do this can be very difficult. This is certainly the case for some student nurses. Our results suggest ways in which educationalists might intervene to support students who witness poor practice to report.

\section{Key words}

Poor practice, reporting, student, cause for concern, nursing

\section{Introduction}

The aim of his paper is to discuss the findings of a small scale qualitative study which explored the factors student nurses take into account when considering how to respond to poor care witnessed on practice placement. While it is reasonable to assume that the great majority of health care is of a good standard, it would be naive to believe that this is always the case, as concerns have been raised about aspects of care across the western world (Atree 2007, Fisher \& Freshwater 2014, Francis 2013, Hazelton et al 2011). Concerns in this context might refer to mistakes and errors as well as cases where care or professional behaviour falls below an acceptable standard as a result of conscious action, neglect, incompetence or abuse. The former are often a focus for local and national patient safety programmes, which emphasise the importance of 'no blame' and the value of learning from critical incidents (Hewitt et al, 2014). These instances of human error are not addressed in the current 
paper. Rather the aim of this study was to explore the latter group of concerns by examining the factors which influence how students respond to poor care or unprofessional behaviour which is a result of conscious action or inaction. The professional requirement to report this type of concern is clear from international nursing guidance (International Council of Nurses 2012), in national regulatory codes (e.g. Nursing Council of New Zealand 2012, Nursing and Midwifery Board of Australia 2008, Nursing and Midwifery Council 2008 \& 2011) and in professional advice (e.g. American Nurses Association 2015), all of which contain statements that make it clear that nurses must act to ensure patient safety and dignity when this is at risk or when quality of care is compromised .

\section{Literature}

Empirical work in this area has tended to focus on registrants and is mainly quantitative, with notable exceptions to the latter being Attree (2007), Jackson et al (2010), and Ohnishi et al (2008). Findings indicate that concerns are not uncommon (Moore \& McAuliffe 2012), that fears about negative consequences influence decisions not to report, that such fears are sometimes justified (Jackson et al. 2010) and that good quality whistleblowing policies can mitigate these fears and increase the likelihood of reporting (Public Concern at Work Survey 2008). There is much here that is helpful in terms understanding why nurses do or do not report concerns. The focus of these studies is, however on registrants and not student nurses. While the similarities between the two groups are obvious, their status in the profession, position within organisations, and relative vulnerability suggest that we should be careful not to assume too much about their experiences of similar circumstances. Moreover, much of the existing work also considers the wider whistleblowing issue of self and peer reporting, which, as noted earlier, is not the focus of the current study.

In a recent literature search, eight empirical papers which dealt to a greater or lesser extent with the reporting of poor practice by student nurses or midwives were identified. These drew on samples of students from Ireland (Begley 2002), the UK (Belafontaine 2010, Cornish \& Jones 2010, Ward 2010, Bradbury Jones et al. 2007a, Randle 2003) the UK and Australia (Levett-Jones \& Lathlean 2009) and the UK and Japan (Bradbury Jones et al. 2007b). 
Begley's (2002) mixed-methods study examined aspects of the experience of student midwives in Ireland as they progressed through their course. She noted the very hierarchical nature of professional relationships and the difficulties that students felt arose as a result of this specifically in relation to raising problems with senior colleagues. In the only study dealing exclusively with factors affecting student decisions to report, Belafontaine (2010) carried out semi-structured interviews with six student nurses. They did not always report concerns citing the student mentor relationship, level and type of support available, personal confidence and fears about failing placement as influencing factors. In their study, Cornish and Jones (2010), conducted focus groups with students to gather information on their experience of compliance with moving and handling policy. Students reported witnessing and participating in poor practice, but also highlighted the difficulties of challenging this, citing their sense of powerlessness and vulnerability as reasons for not doing so. Ward's (2010) study semi-structured interviews with forty nursing and midwifery students also revealed evidence of staff non-compliance with best practice in infection control. Participants were sometimes reluctant to report as they feared that doing so might adversely affect their placement grades or result in other negative repercussions.

Bradbury Jones and colleagues (2007a) used critical incident technique to examine case studies provided by sixty six students in order to understand their experiences of empowerment and disempowerment. Students reported feeling empowered when they felt strong enough to raise concerns. For others a sense of powerlessness left them feeling unable to challenge what they knew to be poor practice. Randle (2003) carried out unstructured interviews with students at the beginning (n $=56)$ and end $(n=39)$ of their degree programme with the aim of examining how their experiences impacted their self-esteem. Amongst descriptions of personal bullying, participants also described how their initial reactions to witnessing poor care and bullying of patients became dulled over time and how they ultimately adopted similar practice as a way of coping.

Levett- Jones and Lathlean (2009) used semi-structured interviews with eighteen Australian and UK students to examine how they negotiated their acceptance into the world of practice placement. They found that students worked hard to fit in and were keen not to make enemies by speaking up, but, as 
self-confidence grew, they felt more able to raise concerns. Finally Bradbury-Jones et al (2007b) used critical incident technique with students from Japan and the UK to compare their experiences of empowerment and disempowerment in practice. They noted that the notion of nurse as patient advocate was not recognised by Japanese students, but was well established in the minds of their UK counterparts, but that they in turn did not always feel empowered to advocate, even when not to do so might lead to potential harm to patients.

In summary, students appear to understand that there is a requirement to report. There is also some limited, evidence that concern about the potential impact on the reporter in terms of future job prospects, academic grades and relationships with others is taken into account when students consider how to respond to witnessing poor care. Students are also mindful of their lowly status in what is perceived to be hierarchical work environment. The outcome of this is that some poor care goes unreported.

In this paper we focus exclusively on examining student explanations of the factors that influence their decisions on whether or not to report poor practice or unprofessional behaviour witnessed while on placement. Our aim is to make a contribution to the wider debate about quality of care, while shedding further light on issues educators may need to consider when preparing students for clinical practice. 


\section{Methods}

\section{Participants}

All pre-registration students on an honours programme $(n=276)$ were invited to take part in the study. Thirteen agreed to participate (P1 to P13) and all were interviewed. This relatively small percentage uptake may reflect one or more of a number of factors, from disinterest in the project to an absence of relevant experience or a fear about perceived repercussions arising from involvement. The age range of participants was from twenty to forty seven with nine females and four males. Of those interviewed, three were training to be mental health nurses, one had withdrawn from this programme at the time of interview and the remaining nine were adult nursing students. Most had witnessed practice that concerned them. All had an expressed interest in the area.

\section{Data Collection and Analysis}

Interview schedules were developed using the guidelines given by Ross (1997). All interviews were recorded and transcribed verbatim. The research focus was on student experiences of reporting or not reporting practice, and the semi-structured interview approach was chosen because the researchers had few pre-set ideas of how participants would describe these. Interviews were designed to elicit information not only on actions and events, but also on perceptions, values and judgments which students felt to be important in relation to reporting concerns. This approach bracketed researcher opinion and experience and is recommended in qualitative research where the type of responses and information provided by the participants is not pre-established, and so the ideas and concepts arising in the interview reflect those which are important to, and representative of, the participant group (Spradley 1979).

Based on a review of the literature, the interview protocol was developed to facilitate explanation of student experience of concerns about practice, actions taken in response to concerns, explanations for actions and how action was facilitated or inhibited by personal, practice and academic factors. (The interview protocol is available from the authors on request). Interviews lasted between 30 and 50 
minutes. The protocol remained standard throughout, however the interviewer was given leave to be participant-led in terms of key aspects of the experience.

Transcripts were analysed following a systematic process using the guidelines laid out by Bryman (2012). Researchers initially familiarised themselves with the content of the transcripts before individually coding sections of text in to meaning units. These were then discussed by the research team and developed into broader thematic categories which best reflected the data. A single member of the team checked the final analysis for category integrity and fit with the data. Examples of each thematic category were then selected from the data for illustration and these are described below.

\section{Ethical considerations}

Ethical approval was granted by the University Ethics Committee. All participants gave their informed consent prior to interview. They were informed that, in the event of a disclosure of risk (or potential risk) of harm to self or others, which had not previously been reported, the participant would be reminded of the importance of following the University's 'Cause for Concern in Clinical Practice' guidance, and also of the support available from the wider University and via professional organisations. Participant confidentiality was assured prior to obtaining of consent although at the same time interviewees were also informed that the University reserved the right to report concerns as appropriate in any cases involving criminal behaviour. Data was stored safely and securely and was accessible to members of the research team only.

\section{Results}

The themes identified represent the issues that participants felt were relevant and important when considering their decisions about reporting concerns. Four themes were identified. One of these: 'I had no choice', was given only as a reason for reporting. Of the remaining three themes: 'consequences for self', and 'living with ambiguity', were used exclusively to describe reasons why 
participants found it difficult to report. The final category, 'being prepared', included factors that facilitated reporting and factors which did not.

\section{I had no choice}

Those who had reported their concerns drew on this theme, which was made up of two related subcategories. The first of these concerned participants' own moral position, that being the internal sense that attempting to remain silent or inactive on the situation was intolerable.

Thinking back on a specific incident, which had brought to a head a number of related concerns, and why she had reported it, one participant remembered:

'... it was disturbing my sleep... it was bothering me even when I wasn't there' (P8 line 430)

For another the case for reporting was equally clear-cut:

'I couldn't believe what I saw, because you don't have to go through a nursing course to know that hitting vulnerable people is a bad thing, it's kind of built into your sense of what's right and wrong' (P9 line 35)

Several participants referred to a moral standpoint, P12, for example, described the process of deciding to report as involving the individual having to:

'...find where you stand morally and then be able to stand up to it' (P12 line 233) 
For some, the decision to report was grounded in personal beliefs and characteristics:

'I think it is a personal thing as well, I like to think I know what is right and wrong' (P6 line 316)

Some participants talked about a build-up of a series of relatively minor events, which culminated in a subsequent report. For example, the overbearing behaviour of a manager toward a member of staff, for one student was the catalyst for her to report. This participant had previously held unexpressed concerns and had been uncertain of what to do in response to these until the following Rubiconcrossing episode occurred:

'When the manager turned on the member of staff in front of me, I felt that was unacceptable. I tried speaking to her about that and I just felt that my concerns were not being taken on board, so at that stage I got in touch with a lecturer'. (P8 line 151).

The second sub-category relates to the ethical issues raised by the relevant professional code of conduct, in this case the NMC Code (NMC, 2008), and other standards or professional guidance. For some students, the professional requirement to protect the public was paramount in their decision to raise concerns. This is summed up by Participant 4 who, when talking about why he had challenged a qualified member of staff about the quality of care being delivered, said:

'It's not will you follow it? [The Code NMC 2008], it's you must follow it. You must make the patient safety your first concern. For me this is these are the standards that guide me in a situation'. (P4 line 318). 
Participant 1, who had worked in care prior to the commencement of training drew on another related explanation for raising her concerns, which reflects how confidence in knowing right from wrong can impact on the propensity to report:

'I had been on training courses about safeguarding vulnerable people and so I was aware of what was bad practice and what was good practice. This made me a bit stronger in terms of reporting things because I had a background of knowing what was deemed to be wrong'. (P1 line 174)

Despite this clarity of thinking in terms of knowing when to report from a personal/moral or professional perspective, those who did so often felt guilty. This appears to be a tension in all the reports where 'doing the right thing' was accompanied in almost all cases by a sense of personal challenge and responsibility for a negative event. The quotation below is illustrative of this.

'I felt like I had done something wrong, I felt horrendous, but then there was another part of me that said, if you don't report this, then something even worse could happen and you knew about it and you didn't say anything' (P1 line 324)

Decisions to report were often accompanied by a degree of reflection and self-doubt. This process of decision-making was extended in some cases and did not necessarily terminate in a report occurring, with some students continuing to reflect on an incident many months and even years after it had occurred. One participant felt sufficiently strongly about her experience that she agreed to participate in this research several years after having transferred to another course. 


\section{Consequences for self}

Students often had an acute awareness of the potential consequences of reporting. These might be both personal and professional. In the extract below an example is given of what happened to a qualified nurse who had previously reported colleagues:

'There was a nurse I use to chat with all the time who was lovely and she said to me one day 'You know I never fit in here because I reported something and now nobody speaks to me because they don't want me grassing (sic) them in'. She would sit on her lunch break by herself and she never had any friends because they knew her as the local tell-tale and I felt so much for her when she told me that'. (P9 line 288)

This participant went on to say:

'If you see somebody doing something wrong and you do the right thing and report it, you can't work there anymore, you just can't because you are never going to be accepted ... without feeling awkward, without the whispering behind your back' (P9 line 312)

Fear of the potential professional consequences of reporting is also very clear in the extract below from an interview with participant 13:

'The biggest thing people are worried about is making a name for themselves by reporting. I have heard staff nurses say that nursing is the bitchiest profession and a lot of us are worried about jobs afterwards. If you make a negative name for yourself it can get around shockingly fast afterwards'. (P13 line 47). 
The concerns of participant 6 focused on the possible impact on the grade she would receive for placement:

'You feel that you might fail,... some people have said, if I stand up for myself, I will fail'. (P6 line 344)

While this student was clearly aware of the potential negative consequences, she was also clear about what she would do in this type of situation:

'If I fail because I have stopped someone getting screamed at, then I don't think that's failing. I would be more than happy to raise that with the University ... I think I would be given enough support [by the University]as other people have been'. (P6 line 350)

Participant 8 summed up this fear of consequences in the following manner:

'It's easy for people to just keep their heads down and get on with it and not raise a concern because of the implications for them. I think a lot of people just don't want to get involved with it. They just think, keep my head down, get through it and carry on'. (P8 line 547)

Non-reporting, or delayed reporting was justified in many cases by arguments of fears, mainly in the abstract, about negative professional and personal consequences. Interestingly, however, none of the 
reports involved first person experiences of negative outcomes, apart from a sense of discomfort or guilt at causing problems for other healthcare professionals whose standards of practice had fallen below an acceptable level.

\section{Living with ambiguity}

While some participants encountered practice which they quickly evaluated as poor and went on to report this, others were faced with situations they found less clear cut, where they called into question their own judgments. Participants often tried to gauge the accuracy of their own perceptions according to the responses of staff members and other students:

'I began to wonder if I was over-reacting ... my mentor just brushed it off and got on with things... it seemed normal to her and also to the auxiliary who was helping'.(P9 line 55)

For another student, the problem related to a difficulty in deciding whether or not the care she witnessed was poor, or if her uneasiness was a consequence of her own lack of experience:

'I don't know if it is a cause for concern or not, but sometimes the way the patients were treated was not how I would treat them'. (P5 line 22)

For one participant the decision not to report poor care was made as a result of her belief that the poor practice she witnessed could be explained by the lack of experience and training provided to care staff: 
'...I never really thought about reporting any of it because it was mostly coming from not knowing any better.' (P10 line 315)

A second sub-theme, emerging from this category, indicated that although events may be clearly worth reporting, the perceived impact of reporting was considered to be negligible; leading to a sense that action was pointless. In this example, the student clearly identified concerns, but explained that she did not report as she believed the clinical area was already known for its poor practice and that it was commonly held, by those with knowledge of the area, that this would not change, regardless of action. Reflecting on a conversation with another nurse the student explained:

'[I was told] that [this] is a unit that runs its own way and is run by a Senior Charge Nurse (SCN) who is laughable. There is no point going any further [with the complaint] because people have done it in the past and the SCN is still there and the practice is still the same. (P11 line 53)

\section{Being prepared}

While all interviewees acknowledged at some level that they were aware of the procedure for raising concerns with the University, there was no clear evidence that knowledge of the process alone was sufficient. Some indicated that more could be done by the University to reassure the potential reporter that they would be protected if they raised concerns:

'...to prepare students it would be useful to make them aware of the protection they will get if they decide to report somebody. It would be very reassuring to know that you would be protected against any unfair treatment.' (P9 line 468) 
This interviewee went on to clarify what she meant in this case by 'protection':

'Something that is more compassionate and that makes you more willing to go forward and report it. Something that will speak to the person inside you that's really scared of being picked on ... you want to know that you are going to be perfectly safe from bullying and being picked on after reporting something'. (P9 467)

A more applied approach to education about raising concerns was thought to be needed and several interviewees suggested that a more reflective and scenario based approach to preparing students to deal with type of event would be helpful. In addition, it was also clear that preparation also involved some form of relationship-building with University staff. For one participant at least, this had not occurred:

'....I feel no connection with them [lecturers]. It's like they don't really know me. I would rather work through something like that with somebody who knows me'. (P11 line 505).

Another recounted how she had approached a member of the teaching team at an early stage in her education and with a relatively small issue, which was unconnected to practice. The student's perception of the staff member's response at that time was that the person was not particularly interested. During the interview she reasoned that a similar response could be expected if she reported poor practice.

The possibility of a negative, or at best disinterested response from the academic team clearly influenced this student's thinking on whether or not to report. 
This contrasted with the actual and very positive experience of a student who did report concerns:

'The University were really, really good.... Really supportive and the policy got followed. I remember feeling really bad for doing it and they were like, don't feel bad. I had emails from them asking if I was ok. I had to be interviewed by the Council and the Care Commission and the Police and they were present at all of these to make sure I wasn't coerced into saying something different... The staff were all aware. They were all really good'. (P1 line 269)

For another student self-confidence was an influencing factor. This appeared to have been built over the course of her time on the programme. Comparing herself as a second year student to how she had been in her first year, participant 3 said:

'I've got a lot more confidence now in that I would be able to stand up to a mentor and ask them to explain what they were doing and why they were doing it' (P3 line 191)

\section{Discussion}

The focus of this study is the reporting of poor practice and unacceptable professional behaviour and, as such, participants talk about a variety of situations of differing severities ranging from the relatively minor (talking over a patient) to the more serious (assault). Four areas were the focus of discussion around decisions on reporting. They were as follows: statements around personal and moral choice, real and potential consequences of action, dealing with the ambiguity of the situation, and being adequately prepared for the reality of reporting. In general the results are consistent with the findings of previous work, but add some detail and depth, while further highlighting an apparent 
gulf between the theoretical expectation, that best practice is the norm and negative exceptions reported, and the realities of practice. Students seem to be negotiating their own position in response to situations, developing a sense of what is acceptable to themselves and balancing this against the expectations of the University, the profession and the sometimes ambiguous messages from fellow students and clinical staff. In all of this there is a real sense in which the student is sometimes alone in a difficult situation. This was particularly apparent in those who reflected on the episodes in which others appeared unconcerned by poor practice or when they considered the potential personal consequences of raising a concern.

From those who did report their concerns there was an acknowledgement that the University was a great source of support. In contrast, the uncertainty of some non-reporters about the likelihood of help highlights the importance of congruence between what academics say and how they actually respond across a range of situations. It should not come as a surprise that inconsistency, even over what might appear to be a trivial issue, may undermine a student's confidence in faculty staff.

Our results indicate that openness is not always facilitated by the work environment, nor is it rewarded. By their nature, the situations students find themselves in are often complex and require significant personal and professional resilience, underpinned by a sound understanding of the professional and ethical framework in which they operate in order to resolve dilemmas in a way that is consistent with public expectations (Edwards 2009). Some of those who were interviewed lacked elements of this preparation and there can be no doubt that educators have a key role to play in developing these. While understanding the process for reporting concerns is important, it is only half the story as students also need to understand why this is so important and why it will sometimes require them to put their heads above the parapet. In light of this, they will also need to know that they will get support when they need it.

In more general terms, our results point to a situation in which some students struggle to engage in the type of robust critical reflection which Roberts and Ion (2014) have argued is an essential corrective 
in modern health care systems, which are so often characterised by habituation to rule governed rationality.

Finally, this work did not set out to report on the prevalence of poor practice witnessed by student nurses on placement and there is currently no literature of this type. It does, however, add to the emerging evidence that poor practice is a problem which may be more widespread than is generally acknowledged.

\section{Limitations}

The findings from this single site, qualitative study may reflect specific local concerns or issues for this group of students. Caution should therefore be exercised before generalising, although it is important to remember that the findings are broadly consistent with the limited literature already available.

\section{Conclusions}

The findings suggest that students have varying levels of awareness regarding their responsibilities in relation to escalating and raising concerns. A clear moral or ethical position appeared to guide those students who decided to report. In contrast, some students appear to see themselves on a journey to registration, which requires them to keep a low profile and simply 'get on with it' regardless of what is happening around. A number of other reasons were given for not reporting. These included concerns about the potential impact on grades, the prospect of interpersonal conflict and an uncertainty about the seriousness of the concern. These factors are all consistent with findings from other studies. The role of academic staff was also raised as it appears that when the student is unsure of the response they will receive from lecturers there is a risk they will not report.

Perhaps one of the most interesting conclusions is that those who reported did so as a result of a commitment to a moral or ethical principle. Strengthening these aspects of the student through education may be one way of ensuring that the practitioners of the future are better equipped to deal with the moral dilemmas that increasingly characterise nursing work. 


\section{References}

American Nurses Association. (2015) Code of Ethics revised version. Retrieved from http://www.nursingworld.org/Mobile/Code-of-Ethic 05/92/15

Attree, M. (2007) Factors influencing nurses' decisions about care quality. Journal of Nursing Management 15, 392-402.

Begley, C. (2002) 'Great fleas have little fleas': Irish students midwives' views of the hierarchy in midwifery. Journal of Advanced Nursing 38 (3) 310 - 317.

Belafontaine, N. (2010) Exploring whether student nurses report poor practice they have witness on placements: An investigation into whether student nurses report poor practice they have witnessed on clinical practice and the factors that influence their decisions. Nursing Times 105 (35) $28-31$.

Bradbury-Jones C., Sambrook S. \& Irvine F. (2007a) The meaning of empowerment for nursing students: a critical incident study. Journal of Advanced Nursing 59 (4) $342-351$.

Bradbury-Jones C., Irvine F.\& Sambrook S. (2007b) Empowerment of nursing students in the United Kingdom and Japan: a cross-cultural study. Journal of Advanced Nursing 35 (4) 379 - 387.

Bryman, A. (2012) Social Research Methods, 4th Ed. Oxford, Oxford University Press..

Cornish J. and Jones A. (2010) Factors affecting compliance with moving and handling policy: Student nurses' views and experiences. Nurse Education in Practice 10 (2) 96 - 100.

Edwards S.D, (2009) Nursing ethics: a principle based approach. 2nd Ed. Basingstoke, Palgrave McMillan.

Fisher P and Freshwater D. (2014) Towards compassionate care through aesthetic rationality. Scandinavian Journal of Caring Science 28, 767-774.

Francis R. (2013) Report of the Mid Staffordshire NHS Foundation Trust Public Inquiry. London, Stationary Office 
Hazelton M, Rossiter R, and Morrall P. (2011) Encounters with the 'dark side': new graduate nurses' experiences in a mental health service, Health Sociology Review (2011) 20(2): 172-186.

Hewitt T, Chreim, S. \& Forster, A. (2014) Sociocultural factors influencing incident reporting among physicians and nurses: understanding frames, underlying self- and peer-reporting practices. Journal of Patient Safety, 0, 0, 1-9.

International Council of Nurses. (2012) ICN Code of conduct for nurses. Retrieved from http://www.icn.ch/images/stories/documents/about/icncode_english.pdf 06/02/15

Jackson D, Peters K, Andrew S, Edenborough M, Halcomb E, Luck L, Salmonsen Y \& Wilkes L. (2010) Understanding whistleblowing: qualitative insights from nurse whisteblowers. Journal of Advanced Nursing, 66 (10), 2194, 2201.

Levett-Jones T. and Lathlean J. (2009) 'Don't rock the boat': Nursing students' experiences of conformity and compliance. Nurse Education Today 29 (3) 342 - 349.

Moore L and McAuliffe E, (2012) To report or not to report: Why some nurses are reluctant to whistleblow. Clinical Governance: An International Journal, 17,4 332-342.

Nursing Council of New Zealand. (2012) Code of conduct. Retrieved from http://www.nursingcouncil.org.nz/Nurses/Code-of-Conduct 05/02/15

Nursing and Midwifery Board of Australia. (2008) Code of ethics for nurses Retrieved from http://www.nursingmidwiferyboard.gov.au/Codes-Guidelines-Statements/Codes-

Guidelines.aspx\#codesofethics 05/02/15

Nursing and Midwifery Council. (2008) The code: standards of conduct, performance and ethics for nurses and midwives. London, NMC

Nursing and Midwifery Council (2010) Standards for pre-registration education. London, Nursing and Midwifery Council. 
Nursing and Midwifery Council (2011) Raising concerns: Guidance for nurses and midwives. Accessed online 15/03/14. http://www.nmc-uk.org/documents/nmc-publications/nmc-raising-andescalating-concerns.pdf

Nursing and Midwifery Council (2013) NMC Response to the Francis Report: the response of the Nursing and Midwifery Council to the Mid Staffordshire NHS Foundation Trust public inquiry report .Retrieved from http://www.nmcuk.org/Documents/Francis\%20report/NMC\%20response\%20to\%20the\%20Francis\% 20report\%2018\%20July.pdf on 16/03/14

Ohnishi, K., Hayama , Y., Asai, A. and Kosugi , S., (2008). The process of whistleblowing in a Japanese psychiatric hospital. Nursing Ethics, 15(5), pp. 631-42.

Public Concern at Work. (2008) Public Concern at Work/Nursing Standard Whistleblowing Survey 2008. Retrieved from http://www.pcaw.co.uk/files/WBsurvey_summary.pdf on 07/01/15

Randle, J. (2003) Bullying in the nursing profession. Journal of Advanced Nursing. 43(4) 395 - 401

Roberts, M. \& Ion, R. (2014) A critical consideration of systemic moral catastrophes in modern health care systems: an Arendtian perspective. Nurse Education Today, 34, 673-675.

Ross, E. (1997) Qualitative Research Methods - data collection and analysis in Research Methods in Primary Care, eds. Carter, Y. \& Thomas, C., Radcliffe Medical Press Ltd, Abingdon.

Spradley, J. (1979) The Ethnographic Interview. New York, Holt, Rhinehart and Winston Ward, D. J. (2010) Infection control in clinical placements: experiences of nursing and midwifery students. Journal of Advanced Nursing. 66 (7) 1533 - 1543. 